\title{
The Convolution and Fractional Derivative of Distributions
}

\author{
Chenkuan Li*, Kyle Clarkson and Vrajna Patel \\ Department of Mathematics and Computer Science, Brandon University, Brandon, Manitoba Canada R7A 6A9 \\ Email: lic@brandonu.ca
}

\begin{abstract}
Let $\left\{\tau_{n}\right\}$ be a certain sequence of functions in $\mathcal{D}$ converging to 1 in $\mathcal{D}^{\prime}$. The commutative neutrix convolution $f \otimes g$ of two distributions $f$ and $g$ in $\mathcal{D}^{\prime}$ is defined to be the neutrix limit of the sequence

$$
\frac{1}{2}\left\{\left(f \tau_{n}\right) * g+f *\left(g \tau_{n}\right)\right\}
$$

provided the limit exists. We present relations between this new convolution and other existing distributional convolutions, and demonstrate its strong computational power in evaluating convolutions as well as applications to defining new fractional derivatives and integrals of generalized functions in the new space $H$ which contains $\mathcal{D}^{\prime}\left(R^{+}\right)$. The neutrix convolutions $x_{-}^{\lambda} \otimes x_{+}^{\mu}$ for $\lambda, \mu, \lambda+\mu \neq 0, \pm 1, \pm 2, \cdots$ and $x_{-}^{\lambda} x_{+}^{s}$ for $\lambda \neq 0, \pm 1, \pm 2, \cdots$ and $s=0,1,2, \cdots$ are evaluated from which other neutrix convolutions are deduced.
\end{abstract}

Keywords: Distribution, convolution, delta function, neutrix limit and fractional derivative.

\section{Introduction}

One of the problems in distribution theory is the lack of definition for convolution and product of distributions in general ([1], [2], [3] and [4]). It is well known that studying fractional calculus of distributions and many types of integral equations are based on distributional convolutions ([5], [6], [7], [8] and [9]). The classical definition for the convolution of two functions $f$ and $g$ is as follows:

Definition 1.1 Let $f$ and $g$ be functions. Then the convolutional product $f * g$ is defined by

$$
(f * g)(x)=\int_{-\infty}^{\infty} f(t) g(x-t) d t
$$

if the integral exists.

Let $\mathcal{D}$ be the Schwartz space [9] of infinitely differentiable functions with compact support in $R$, and $\mathcal{D}^{\prime}$ be the space of distributions (linearly continuous functionals) defined on $\mathcal{D}$. Further, we shall define a sequence $\phi_{1}(x), \phi_{2}(x), \cdots, \phi_{n}(x), \cdots$ which converges to zero in $\mathcal{D}$ if all these functions vanish outside a certain fixed and bounded interval, and converge uniformly to zero (in the usual sense) together with their derivatives of any order. The functional $\delta$ is defined as

$$
(\delta, \phi)=\phi(0)
$$

where $\phi \in \mathcal{D}$. Clearly, $\delta$ is a linear and continuous functional on $\mathcal{D}$, and hence $\delta \in \mathcal{D}^{\prime}$.

The convolution of certain pairs of distributions is usually defined as follows, see Gel'fand and Shilov [9] for example.

Definition 1.2 Let $f$ and $g$ be distributions in $\mathcal{D}^{\prime}$ satisfying either of the following conditions:

(a) either $f$ or $g$ has bounded support (set of all essential points), or

(b) the supports of $f$ and $g$ are bounded on the same side.

Then the convolution $f * g$ is defined by the equation

$$
((f * g)(x), \phi(x))=(g(x),(f(y), \phi(x+y)))
$$

for $\phi \in \mathcal{D}$. 
Note that if $f$ and $g$ are locally integrable functions satisfying either of the conditions in (a) or (b) in Definition 1.2, then Definition 1.2 is in agreement with Definition 1.1. It also follows that if the convolution $f * g$ exists by Definition 1.1 or 1.2 , then the following equations hold:

$$
\begin{aligned}
& f * g=g * f \\
& (f * g)^{\prime}=f * g^{\prime}=f^{\prime} * g
\end{aligned}
$$

where all the derivatives above are in the distributional sense.

Definitions 1.1 and 1.2 are very restrictive and can only be used for a small class of distributions. In order to extend the convolution to a larger class of distributions, Jones [10] introduced the following definition.

Definition 1.3 Let $f$ and $g$ be distributions in $\mathcal{D}^{\prime}$ and let $\tau(x)$ be an infinitely differentiable function satisfying the following conditions:

(i) $\tau(x)=\tau(-x)$,

(ii) $0 \leq \tau(x) \leq 1$,

(iii) $\tau(x)=1$ if $|x| \leq 1 / 2$,

(iv) $\tau(x)=0$ if $|x| \geq 1$.

Let

$$
f_{n}(x)=f(x) \tau(x / n), \quad g_{n}(x)=g(x) \tau(x / n)
$$

for $n=1,2, \cdots$. Then the convolution $f * g$ is defined as the limit of the sequence $\left\{f_{n} * g_{n}\right\}$, provided the limit $h$ exists in the sense that

$$
\lim _{n \rightarrow \infty}\left(f_{n} * g_{n}, \phi\right)=(h, \phi)
$$

for all testing functions $\phi \in \mathcal{D}$.

The convolution $f_{n} * g_{n}$ in Definition 1.3 clearly exists by Definition 1.2 as $f_{n}$ and $g_{n}$ have bounded support. Furthermore, equation (1) holds. However, equation (2) need not necessarily hold since Jones [10] proved that

$$
\begin{aligned}
& 1 * \operatorname{sgn} x=\operatorname{sgn} x * 1=x, \\
& (1 * \operatorname{sgn} x)^{\prime}=1, \quad 1^{\prime} * \operatorname{sgn} x=0,1 *(\operatorname{sgn} x)^{\prime}=1 * 2 \delta(x)=2 .
\end{aligned}
$$

It can be proven that if a convolution exists by Definition 1.1 or 1.2 then it exists by Definition 1.3 and defines the same distribution. Therefore, Definition 1.3 generalizes Definitions 1.1 and 1.2. However, there are still many convolutions which cannot be given by Definition 1.3. In order to fix this, Fisher [11] presented the following definition.

Definition 1.4 Let $f$ and $g$ be distributions and let

$$
\tau_{n}(x)= \begin{cases}1 & \text { if }|x| \leq n, \\ \tau\left(n^{n} x-n^{n+1}\right) & \text { if } x>n, \\ \tau\left(n^{n} x+n^{n+1}\right) & \text { if } x<-n,\end{cases}
$$

for $n=1,2, \cdots$, where $\tau$ is defined as in Definition 1.3. Let $f_{n}(x)=f(x) \tau_{n}(x)$ for $n=1,2, \cdots$. Then the noncommutative neutrix convolution $f \circledast g$ is defined as the neutrix limit of the sequence $\left\{f_{n} * g\right\}$, provided the limit $h$ exists in the sense that

$$
N-\lim _{n \rightarrow \infty}\left(f_{n} * g, \phi\right)=(h, \phi)
$$

for all $\phi \in \mathcal{D}$, where $N$ is the neutrix, see van der Corput [12] (use the neutrix to abandon unwanted infinite quantities from asymptotic expressions), having domain $N^{\prime}=\{1,2, \cdots\}$ and range the real numbers, with negligible functions that are finite linear sums of functions

$$
n^{\lambda} \ln ^{r-1} n, \quad \ln ^{r} n, \quad(\lambda>0, r=1,2, \cdots)
$$

and all functions of $n$ that converge to zero in the normal sense as $n$ tends to infinity. 
The convolution $f_{n} * g$ in this definition is again in the sense of Definition 1.2 as the support of $f_{n}$ is contained in the interval $\left[-n-n^{-n}, n+n^{-n}\right]$. It is also proven that if a convolution exists by Definition 1.1 or 1.2 then the noncommutative neutrix convolution exists and defines the same distribution [11].

To overcome the convolutional noncommutativity above, Fisher and Li [13] introduced the following commutative neutrix convolution of distributions $f$ and $g$ by $f * g$ to distinguish it from the noncommutative neutrix convolution in Definition 1.4.

Definition 1.5 Let $f$ and $g$ be distributions and let $\tau_{n}$ be defined as in Definition 1.4. Let $f_{n}(x)=f(x) \tau_{n}(x)$ and $g_{n}(x)=g(x) \tau_{n}(x)$ for $n=1,2, \cdots$. Then the commutative neutrix convolution $f * g$ is defined as the neutrix limit of the sequence $\left\{f_{n} * g_{n}\right\}$, provided the limit $h$ exists in the sense that

$$
N-\lim _{n \rightarrow \infty}\left(f_{n} * g_{n}, \phi\right)=(h, \phi)
$$

for all $\phi \in \mathcal{D}$, where $N$ is the neutrix given above.

\section{The New Commutative Neutrix Convolution}

Let $\delta_{n}(x)=n \rho(n x)$ be Temples' $\delta$-sequence for $n=1,2, \cdots$, where $\rho(x)$ is a fixed, infinitely differentiable function on $\mathrm{R}$ with the four properties:

(i) $\rho(x) \geq 0$,

(ii) $\rho(x)=0$ for $|x| \geq 1$,

(iii) $\rho(x)=\rho(-x)$,

(iv) $\int_{-\infty}^{\infty} \rho(x) d x=1$.

Both Kilicman [14] and $\mathrm{Li}$ [15], in 2001 and 2007 respectively, used the following distributional product definition to deduce several commutative products.

Definition 2.1 Let $f$ and $g$ be distributions and let $\tilde{f}_{n}=f * \delta_{n}$ and $\tilde{g}_{n}=g * \delta_{n}$. We say that the commutative neutrix product $f \diamond g$ of $f$ and $g$ exists and is equal to $h$ if

$$
N-\lim _{n \rightarrow \infty} \frac{1}{2}\left\{\left(\tilde{f}_{n} g, \phi\right)+\left(f \tilde{g}_{n}, \phi\right)\right\}=(h, \phi)
$$

for all testing functions $\phi \in \mathcal{D}$. If the normal limit exists, then it is simply called the commutative product. Note that $\tilde{f}_{n}$ and $\tilde{g}_{n}$ are two infinitely differentiable functions and hence $\left(\tilde{f}_{n} g, \phi\right)$ as well as $\left(f \tilde{g}_{n}, \phi\right)$ are well defined.

As suggested from this definition, we present the following commutative neutrix convolutional definition.

Definition 2.2 Let $f$ and $g$ be distributions and let $\tau_{n}$ be defined as in Definition 1.4. Let $f_{n}(x)=f(x) \tau_{n}(x)$ and $g_{n}(x)=g(x) \tau_{n}(x)$ for $n=1,2, \cdots$. Then the commutative neutrix convolution $f \propto g$ of $f$ and $g$ exists and is equal to $h$ if

$$
N-\lim _{n \rightarrow \infty} \frac{1}{2}\left\{\left(\left(f \tau_{n}\right) * g, \phi\right)+\left(f *\left(g \tau_{n}\right), \phi\right)\right\}=(h, \phi)
$$

for all testing functions $\phi \in \mathcal{D}$. If the normal limit exists, then it is simply called the commutative convolution. Clearly, this definition generalizes Definitions 1.1 and 1.2.

Theorem 2.1 The commutative convolution $x^{\lambda}\left(\operatorname{sgn} x \cdot x^{\lambda}\right)$ exists and

$$
x^{\lambda} \otimes\left(\operatorname{sgn} x \cdot x^{\lambda}\right)=\frac{\Gamma^{2}(\lambda+1)}{\Gamma(2 \lambda+2)} x^{2 \lambda+1}
$$

for $\lambda>-1$.

Proof. Consider the sum

$$
\begin{aligned}
& I=\left(x^{\lambda} \tau_{n}(x)\right) *\left(\operatorname{sgn} x \cdot x^{\lambda}\right)+\left(\operatorname{sgn} x \cdot x^{\lambda} \tau_{n}(x)\right) * x^{\lambda} \\
& =\int_{-\infty}^{\infty} t^{\lambda} \tau_{n}(t) \operatorname{sgn}(x-t)(x-t)^{\lambda} d t+\int_{-\infty}^{\infty} \operatorname{sgn} t t^{\lambda} \tau_{n}(t)(x-t)^{\lambda} d t \\
& =I_{1}+I_{2} .
\end{aligned}
$$


Clearly, for $x \geq 0$

$$
\begin{aligned}
& I_{1}=\int_{-\infty}^{0} t^{\lambda} \tau_{n}(t)(x-t)^{\lambda} d t+\int_{0}^{x} t^{\lambda} \tau_{n}(t)(x-t)^{\lambda} d t-\int_{x}^{\infty} t^{\lambda} \tau_{n}(t)(x-t)^{\lambda} d t, \\
& I_{2}=-\int_{-\infty}^{0} t^{\lambda} \tau_{n}(t)(x-t)^{\lambda} d t+\int_{0}^{x} t^{\lambda} \tau_{n}(t)(x-t)^{\lambda} d t+\int_{x}^{\infty} t^{\lambda} \tau_{n}(t)(x-t)^{\lambda} d t .
\end{aligned}
$$

This implies that

$$
I=I_{1}+I_{2}=2 \int_{0}^{x} t^{\lambda} \tau_{n}(t)(x-t)^{\lambda} d t
$$

which is also true for $x<0$ by a similar argument. Therefore,

$$
\begin{aligned}
\left(x^{\lambda}\left(\operatorname{sgn} x \cdot x^{\lambda}\right), \phi\right) & =\lim _{n \rightarrow \infty}\left(\int_{0}^{x} t^{\lambda} \tau_{n}(t)(x-t)^{\lambda} d t, \phi\right) \\
& =\left(\int_{0}^{x} t^{\lambda}(x-t)^{\lambda} d t, \phi\right)
\end{aligned}
$$

since $\phi \in \mathcal{D}$ and $\tau_{n}(t)=1$ for $|t| \leq n$.

The conclusion follows immediately from

$$
\int_{0}^{x} t^{\lambda}(x-t)^{\lambda} d t=B(\lambda+1, \lambda+1) x^{2 \lambda+1}=\frac{\Gamma^{2}(\lambda+1)}{\Gamma(2 \lambda+2)} x^{2 \lambda+1}
$$

where $B$ denotes the Beta function. This completes the proof of Theorem 2.1.

In particular, we come to

$$
x^{r}\left(\operatorname{sgn} x \cdot x^{r}\right)=\frac{(r !)^{2}}{(2 r+1) !} x^{2 r+1}
$$

for $r=0,1,2, \cdots$. This completely coincides with Fisher's result in [16] by Definition 1.3.

\section{Theorem 2.2}

$$
\begin{aligned}
& \left(\operatorname{sgn} x \cdot x^{r}\right) \circledast x^{r}=0, \\
& x^{r} \circledast\left(\operatorname{sgn} x \cdot x^{r}\right)=\frac{2(r !)^{2}}{(2 r+1) !} x^{2 r+1}
\end{aligned}
$$

for any nonnegative integer $r$.

Proof. We have

$$
\begin{aligned}
\left(\operatorname{sgn} x \cdot x^{r} \tau_{n}(x)\right) * x^{r}= & \int_{-\infty}^{\infty} \operatorname{sgnt} t^{r} \tau_{n}(t)(x-t)^{r} d t \\
= & -\int_{-\infty}^{0} t^{r} \tau_{n}(t)(x-t)^{r} d t+\int_{0}^{\infty} t^{r} \tau_{n}(t)(x-t)^{r} d t \\
= & -\int_{-n-n^{-n}}^{-n} t^{r} \tau_{n}(t)(x-t)^{r} d t-\int_{-n}^{0} t^{r}(x-t)^{r} d t+\int_{0}^{n} t^{r}(x-t)^{r} \\
& +\int_{n}^{n+n^{-n}} t^{r} \tau_{n}(t)(x-t)^{r} d t \\
= & I_{1}+I_{2}+I_{3}+I_{4} .
\end{aligned}
$$

Applying

$$
(x-t)^{r}=(-1)^{r} \sum_{i=0}^{r}\left(\begin{array}{l}
r \\
i
\end{array}\right)(-1)^{i} x^{i} t^{r-i}
$$


we get

$$
\lim _{n \rightarrow \infty}\left(I_{1}, \phi\right)=(-1)^{r+1} \sum_{i=0}^{r}\left(\begin{array}{l}
r \\
i
\end{array}\right)(-1)^{i} \lim _{n \rightarrow \infty} \int_{-n-n^{-n}}^{-n} \tau_{n}(t) t^{2 r-i} d t \int_{-\infty}^{\infty} x^{i} \phi(x) d x=0
$$

by noting that $\tau_{n}(t)$ is bounded and $\phi \in \mathcal{D}$. Similarly, we can imply

$$
\lim _{n \rightarrow \infty}\left(I_{4}, \phi\right)=0
$$

Clearly,

$$
N-\lim _{n \rightarrow \infty}\left(I_{2}, \phi\right)=(-1)^{r+1} \sum_{i=0}^{r}\left(\begin{array}{l}
r \\
i
\end{array}\right)(-1)^{i}\left(N-\lim _{n \rightarrow \infty}\right) \int_{-n}^{0} t^{2 r-i} d t \int_{-\infty}^{\infty} x^{i} \phi(x) d x=0 .
$$

Similarly,

$$
N-\lim _{n \rightarrow \infty}\left(I_{3}, \phi\right)=0
$$

Therefore, $\left(\operatorname{sgn} x \cdot x^{r}\right) \circledast x^{r}=0$.

As for the second statement, we have

$$
\begin{aligned}
x^{r} \tau_{n}(x) *\left(\operatorname{sgn} x \cdot x^{r}\right) & =\int_{-\infty}^{\infty} t^{r} \tau_{n}(t) \operatorname{sgn}(x-t)(x-t)^{r} d t \\
& =\int_{-n-n^{-n}}^{x} t^{r} \tau_{n}(t)(x-t)^{r} d t-\int_{x}^{n+n^{-n}} t^{r} \tau_{n}(t)(x-t)^{r} d t=I_{1}+I_{2} .
\end{aligned}
$$

Clearly, we get

$$
\begin{aligned}
\left(I_{1}, \phi\right) & =\left(\int_{-n-n^{-n}}^{-n} t^{r} \tau_{n}(t)(x-t)^{r} d t, \phi\right)+\left(\int_{-n}^{x} t^{r}(x-t)^{r} d t, \phi\right) \\
& =\left(\int_{-n-n^{-n}}^{-n} t^{r} \tau_{n}(t)(x-t)^{r} d t, \phi\right)+\left(\int_{-n}^{0} t^{r}(x-t)^{r} d t, \phi\right)+\left(\int_{0}^{x} t^{r}(x-t)^{r} d t, \phi\right)
\end{aligned}
$$

for $x$ belonging to the support of $\phi$ which is contained in $[-n, n]$.

It follows from the above that

$$
N-\lim _{n \rightarrow \infty}\left(I_{1}, \phi\right)=\left(\int_{0}^{x} t^{r}(x-t)^{r} d t, \phi\right)=\left(\frac{(r !)^{2}}{(2 r+1) !} x^{2 r+1}, \phi\right) .
$$

By analogous arguments, we can prove that

$$
N-\lim _{n \rightarrow \infty}\left(I_{2}, \phi\right)=\left(\int_{0}^{x} t^{r}(x-t)^{r} d t, \phi\right)=\left(\frac{(r !)^{2}}{(2 r+1) !} x^{2 r+1}, \phi\right) .
$$

Hence,

$$
x^{r} \circledast\left(\operatorname{sgn} x \cdot x^{r}\right)=\frac{2(r !)^{2}}{(2 r+1) !} x^{2 r+1} .
$$

This completes the proof of Theorem 2.2.

Remark 1 It is very interesting to point out that both $\left(\left(f \tau_{n}\right) * g, \phi\right)$ and $\left(f *\left(g \tau_{n}\right), \phi\right)$ could be divergent for some distributions $f$ and $g$ in the normal sense as $n$ tends to infinity, but the addition in Definition 2.2 is convergent and easily evaluated due to cancelations, as indicated in Theorem 2.1. Furthermore, it seems much harder to find out the noncommutative convolutions $\left(\operatorname{sgn} x \cdot x^{\lambda}\right) \circledast x^{\lambda}$ and $x^{\lambda} \circledast\left(\operatorname{sgn} x \cdot x^{\lambda}\right)$ for $\lambda>-1$ if it is still possible. On the other hand, one may need to use the neutrix limit to evaluate some distributional convolutions using Definition 2.2 while Definition 1.4 does not do so. The following is an example to demonstrate this. 
By Definition 2.2, we have

$$
x^{2 r} \otimes \operatorname{sgn} x=\frac{x^{2 r+1}}{2 r+1}
$$

for $r=0,1,2, \cdots$. Indeed, we have

$$
\begin{aligned}
& x^{2 r} \tau_{n}(x) * \operatorname{sgn} x+x^{2 r} * \operatorname{sgn} x \tau_{n}(x)=\int_{-\infty}^{\infty} t^{2 r} \tau_{n}(t) \operatorname{sgn}(x-t) d t+\int_{-\infty}^{\infty} \operatorname{sgn} t \tau_{n}(t)(x-t)^{2 r} d t \\
& =\int_{-n-n^{-n}}^{x} t^{2 r} \tau_{n}(t) d t-\int_{x}^{n+n^{-n}} t^{2 r} \tau_{n}(t) d t-\int_{-n-n^{-n}}^{0}(x-t)^{2 r} \tau_{n}(t) d t \\
& \quad+\int_{0}^{n+n^{-n}}(x-t)^{2 r} \tau_{n}(t) d t=I_{1}+I_{2}+I_{3}+I_{4}
\end{aligned}
$$

for $x$ belonging to the support of $\phi$ which is contained in $[-n, n]$.

Clearly,

$$
\begin{aligned}
I_{1}+I_{2} & =\int_{-n-n^{-n}}^{-n} t^{2 r} \tau_{n}(t) d t+\int_{-n}^{x} t^{2 r} d t-\int_{x}^{n} t^{2 r} d t-\int_{n}^{n+n^{-n}} t^{2 r} \tau_{n}(t) d t \\
& =\frac{2 x^{2 r+1}}{2 r+1}
\end{aligned}
$$

by noting that $\tau_{n}(t)$ is an even function. Without the neutrix limit, this derives that

$$
x^{2 r} \circledast \operatorname{sgn} x=\frac{2 x^{2 r+1}}{2 r+1}, \quad r=0,1,2, \cdots
$$

which is identical with the result in [17].

As for $I_{3}+I_{4}$, we have

$$
\begin{aligned}
I_{3}+I_{4}= & -\int_{-n-n^{-n}}^{-n}(x-t)^{2 r} \tau_{n}(t) d t-\int_{-n}^{0}(x-t)^{2 r} d t+\int_{0}^{n}(x-t)^{2 r} d t \\
& \quad+\int_{n}^{n+n^{-n}}(x-t)^{2 r} \tau_{n}(t) d t \\
= & \int_{n}^{n+n^{-n}}\left[(x-t)^{2 r}-(x+t)^{2 r}\right] \tau_{n}(t) d t+\frac{2 x^{2 r+1}}{2 r+1}-\frac{(x+n)^{2 r+1}}{2 r+1}-\frac{(x-n)^{2 r+1}}{2 r+1} .
\end{aligned}
$$

Hence we come to, for $\phi \in \mathcal{D}$,

$$
\begin{aligned}
& \lim _{n \rightarrow \infty}\left(\int_{n}^{n+n^{-n}}\left[(x-t)^{2 r}-(x+t)^{2 r}\right] \tau_{n}(t) d t, \phi\right)=0, \\
& N-\lim _{n \rightarrow \infty}\left(\frac{2 x^{2 r+1}}{2 r+1}-\frac{(x+n)^{2 r+1}}{2 r+1}-\frac{(x-n)^{2 r+1}}{2 r+1}, \phi\right)=0
\end{aligned}
$$

by the binomial formula and noting that

$$
\begin{aligned}
& N-\lim _{n \rightarrow \infty}\left(-\frac{(x+n)^{2 r+1}}{2 r+1}, \phi\right)=\frac{-x^{2 r+1}}{2 r+1}, \\
& N-\lim _{n \rightarrow \infty}\left(-\frac{(x-n)^{2 r+1}}{2 r+1}, \phi\right)=\frac{-x^{2 r+1}}{2 r+1} .
\end{aligned}
$$

Hence

$$
x^{2 r} \operatorname{sgn} x=\frac{x^{2 r+1}}{2 r+1} .
$$


The sum $I_{3}+I_{4}$ in the above also deduces that

$$
\operatorname{sgn} x \circledast x^{2 r}=0, \quad r=0,1,2, \cdots
$$

with the neutrix limit.

Remark 2 Without the neutrix limit, Definitions 1.3 and 1.4 are independent in terms of existence. Indeed, the noncommutative convolution of $x \operatorname{sgn} x$ and $x$ in Definition 1.4 is divergent in the normal sense as indicated above but Fisher proved the following using Definition 1.3 [16]

$$
\left(\operatorname{sgn} x \cdot x^{r}\right) * x^{r}=\frac{(r !)^{2}}{(2 r+1) !} x^{2 r+1}, \quad r=0,1,2, \cdots .
$$

Conversely, $x^{2 r+1} \circledast 1=0$. However, $x * 1$ is divergent in Definition 1.3. In fact,

$$
(x * 1, \phi)=\lim _{n \rightarrow \infty}(x \tau(x / n) * \tau(x / n), \phi) .
$$

Evidently,

$$
x \tau(x / n) * \tau(x / n)=n^{2} \int_{-1}^{1} y \tau(y) \tau(x / n-y) d y .
$$

By the mean value theorem,

$$
\lim _{n \rightarrow \infty} n \int_{-1}^{1} y \tau(y) \tau(x / n-y) d y=-x \int_{-1}^{1} \tau^{\prime}(y) y \tau(y) d y .
$$

for $x$ in the support of $\phi$. Choosing $\phi$ such that $\int_{-\infty}^{\infty} x \phi(x) d x \neq 0$, we come to

$$
(x * 1, \phi)=\lim _{n \rightarrow \infty}-n \int_{-1}^{1} \tau^{\prime}(y) y \tau(y) d y \int_{-\infty}^{\infty} x \phi(x) d x=\infty
$$

by noting that

$$
\int_{-1}^{1} \tau^{\prime}(y) y \tau(y) d y=\frac{1}{2} \int_{-1}^{1} \tau^{2}(y) d y
$$

using integration by parts.

Remark 3 At this moment, it is not quite clear if Definition 1.5 is equivalent to Definition 2.2, although both define the commutative neutrix convolutions. In fact,

$$
\begin{aligned}
& N-\lim _{n \rightarrow \infty} \frac{1}{2}\left\{\left(\left(f \tau_{n}\right) * g, \phi\right)+\left(f *\left(g \tau_{n}\right), \phi\right)\right\}-N-\lim _{n \rightarrow \infty}\left(f_{n} * g_{n}, \phi\right) \\
& =N-\lim _{n \rightarrow \infty} \frac{1}{2}\left\{\left(\left(f \tau_{n}\right) *\left(g-g \tau_{n}\right), \phi\right)+\left(\left(f-f \tau_{n}\right) *\left(g \tau_{n}\right), \phi\right)\right\} .
\end{aligned}
$$

One can neither prove the above being zero nor non-zero by a counter example. The authors welcome and appreciate any discussion from interested readers. However, the computational complexity of Definition 2.2 is less than that of Definition 1.5 in general, since the convolutions in Definition 2.2 only involve one of factors $\tau_{n}(t)$ and $\tau_{n}(x-t)$, while Definition 1.5 has the product $\tau_{n}(t) \tau_{n}(x-t)$ to consider. The following is an example to illustrate this.

By Definition 1.5, we can show

$$
1 *(\operatorname{sgn} x \cdot x)=x^{2} / 2 .
$$

To evaluate this convolution, we need consider

$$
\begin{aligned}
& \int_{-\infty}^{\infty} \operatorname{sgnt} \tau_{n}(t) \tau_{n}(x-t) d t=-\int_{-n-n^{-n}}^{0} t \tau_{n}(t) \tau_{n}(x-t) d t+\int_{0}^{n+n^{-n}} t \tau_{n}(t) \tau_{n}(x-t) d t \\
& =-\int_{-n-n^{-n}}^{-n} t \tau_{n}(t) \tau_{n}(x-t) d t-\int_{-n}^{0} t \tau_{n}(x-t) d t+\int_{0}^{n} t \tau_{n}(x-t) d t \\
& \quad+\int_{n}^{n+n^{-n}} t \tau_{n}(t) \tau_{n}(x-t) d t=I_{1}+I_{2}+I_{3}+I_{4} .
\end{aligned}
$$


Clearly,

$$
\lim _{n \rightarrow \infty} I_{1}=\lim _{n \rightarrow \infty} I_{4}=0
$$

by $\left|\tau_{n}(t) \tau_{n}(x-t)\right| \leq 1$.

As for $I_{2}$, we have for $x<0$ in the support of $\phi$

$$
I_{2}=-\int_{-n}^{0} t \tau_{n}(x-t) d t=-\int_{-n}^{0} t d t=n^{2} / 2
$$

since $\tau_{n}(x-t)=1$.

When $x \geq 0$,

$$
\begin{aligned}
I_{2} & =-\int_{-n}^{0} t \tau_{n}(x-t) d t=\int_{x+n}^{x}(x-y) \tau_{n}(y) d y=-\int_{x}^{n}(x-y) d y-\int_{n}^{n+n^{-n}}(x-y) \tau_{n}(y) d y \\
& =\frac{(x-n)^{2}}{2}-\int_{n}^{n+n^{-n}}(x-y) \tau_{n}(y) d y .
\end{aligned}
$$

Hence,

$$
N-\lim _{n \rightarrow \infty}\left(I_{2}, \phi\right)= \begin{cases}\left(x^{2} / 2, \phi\right) & \text { if } x \geq 0 \\ 0 & \text { if } x<0\end{cases}
$$

Let us compute $I_{3}$ for $x \geq 0$,

$$
I_{3}=\int_{0}^{n} t \tau_{n}(x-t) d t=\int_{x-n}^{x}(x-y) \tau_{n}(y) d y=\int_{x-n}^{x}(x-y) d y=n^{2} / 2
$$

since $\tau_{n}(y)=1$ on $[x-n, x]$, where $x$ is in the support of $\phi$.

When $x<0$,

$$
\begin{aligned}
I_{3} & =\int_{x-n}^{x}(x-y) \tau_{n}(y) d y=\int_{-n-n^{-n}}^{-n}(x-y) \tau_{n}(y) d y+\int_{-n}^{x}(x-y) d y \\
& =(x+n)^{2} / 2+\int_{-n-n^{-n}}^{-n}(x-y) \tau_{n}(y) d y .
\end{aligned}
$$

Therefore,

$$
N-\lim _{n \rightarrow \infty}\left(I_{3}, \phi\right)= \begin{cases}0 & \text { if } x \geq 0 \\ \left(x^{2} / 2, \phi\right) & \text { if } x<0\end{cases}
$$

by noting that

$$
\lim _{n \rightarrow \infty} \int_{-n-n^{-n}}^{-n}(x-y) \tau_{n}(y) d y=0
$$

This completes the proof of equation (4).

We can derive the convolution of 1 and $\operatorname{sgn} x \cdot x$ by Definition 2.2 in the following simpler process as every term only contains one of two factors $\tau_{n}(t)$ and $\tau_{n}(x-t)$. Clearly, 


$$
\begin{aligned}
& \tau_{n}(x) *(\operatorname{sgn} x \cdot x)+1 *\left(\operatorname{sgn} x \cdot x \tau_{n}(x)\right)=\int_{-\infty}^{\infty} \tau_{n}(t) \operatorname{sgn}(x-t) \cdot(x-t) d t+\int_{-\infty}^{\infty} \tau_{n}(t) \operatorname{sgn} t \cdot t d t \\
& =\int_{-\infty}^{x} \tau_{n}(t) \cdot(x-t) d t-\int_{x}^{\infty} \tau_{n}(t) \cdot(x-t) d t+2 \int_{0}^{\infty} t \tau_{n}(t) d t \\
& =\int_{-n-n^{-n}}^{-n} \tau_{n}(t) \cdot(x-t) d t+\int_{-n}^{x}(x-t) d t-\int_{x}^{n}(x-t) d t-\int_{n}^{n+n^{-n}} \tau_{n}(t) \cdot(x-t) d t \\
& \quad+2 \int_{0}^{n} t d t+2 \int_{n}^{n+n^{-n}} \tau_{n}(t) \cdot t d t \\
& =\int_{-n-n^{-n}}^{-n} \tau_{n}(t) \cdot(x-t) d t+(x+n)^{2} / 2+(x-n)^{2} / 2-\int_{n}^{n+n^{-n}} \tau_{n}(t) \cdot(x-t) d t \\
& \quad+n^{2}+2 \int_{n}^{n+n^{-n}} \tau_{n}(t) \cdot t d t .
\end{aligned}
$$

This implies that

$1 \otimes \operatorname{sgn} x \cdot x=x^{2} / 2$.

Theorem 2.3 The commutative neutrix convolution $x_{-}^{\lambda} \otimes x_{+}^{\mu}$ exists and

$$
x_{-}^{\lambda} x_{+}^{\mu}=B(-\lambda-\mu-1, \mu+1) x_{-}^{\lambda+\mu+1}+B(-\lambda-\mu-1, \lambda+1) x_{+}^{\lambda+\mu+1}
$$

for $\lambda, \mu, \lambda+\mu \neq 0, \pm 1, \pm 2, \cdots$, where $B$ denotes the Beta function.

Proof. We will first of all suppose $\lambda, \mu>-1$, so that $x_{-}^{\lambda}$ and $x_{+}^{\mu}$ are locally integrable functions. Consider

$$
\begin{aligned}
\left(\left(x_{-}^{\lambda} \tau_{n}(x)\right) * x_{+}^{\mu}, \phi\right)= & \left(y_{-}^{\lambda} \tau_{n}(y),\left(x_{+}^{\mu}, \phi(x+y)\right)\right) \\
= & \int_{-n-n^{-n}}^{0}(-y)^{\lambda} \tau_{n}(y) \int_{-\infty}^{\infty} x_{+}^{\mu} \phi(x+y) d x d y \\
= & \int_{-n-n^{-n}}^{0}(-y)^{\lambda} \tau_{n}(y) \int_{a}^{b}(x-y)_{+}^{\mu} \phi(x) d x d y \\
= & \int_{a}^{b} \phi(x) \int_{-n-n^{-n}}^{-n}(-y)^{\lambda} \tau_{n}(y)(x-y)_{+}^{\mu} d y d x \\
& +\int_{a}^{b} \phi(x) \int_{-n}^{0}(-y)^{\lambda}(x-y)_{+}^{\mu} d y d x=I_{1}+I_{2}
\end{aligned}
$$

for any testing function $\phi \in \mathcal{D}$ with the support in the interval $[a, b]$.

When $x<0$ and $-n \leq y \leq 0$, we have

$$
\int_{-n}^{0}(-y)^{\lambda}(x-y)_{+}^{\mu} d y=\int_{-n}^{x}(-y)^{\lambda}(x-y)^{\mu} d y .
$$

On making the substitution $y=x u^{-1}$, we arrive at

$$
\int_{-n}^{x}(-y)^{\lambda}(x-y)^{\mu} d y=(-x)^{\lambda+\mu+1} \int_{-x / n}^{1} u^{-\lambda-\mu-2}(1-u)^{\mu} d u .
$$

It follows that

$$
N-\lim _{n \rightarrow \infty} \int_{-n}^{0}(-y)^{\lambda}(x-y)_{+}^{\mu} d y=B(-\lambda-\mu-1, \mu+1)(-x)^{\lambda+\mu+1}
$$


see [18] or Gel'fand and Shilov [9].

When $x>0$, we make the substitution $y=x\left(1-u^{-1}\right)$ to get

$$
\int_{-n}^{0}(-y)^{\lambda}(x-y)^{\mu} d y=x^{\lambda+\mu+1} \int_{x / x+n}^{1} u^{-\lambda-\mu-2}(1-u)^{\lambda} d u .
$$

It follows similarly that

$$
N-\lim _{n \rightarrow \infty} \int_{-n}^{0}(-y)^{\lambda}(x-y)^{\mu} d y=B(-\lambda-\mu-1, \lambda+1) x^{\lambda+\mu+1} .
$$

Furthermore, we can see that

$$
\lim _{n \rightarrow \infty} I_{1}=0
$$

as $\left|(-y)^{\lambda} \tau_{n}(y)(x-y)_{+}^{\mu}\right| \leq\left|\left(n+n^{-n}\right)^{\lambda+2}\left(b+n+n^{-n}\right)^{\mu+2}\right|=O\left(n^{\mu+\lambda+4}\right)$, assuming $b>0$.

In summary,

$$
\begin{aligned}
N-\lim _{n \rightarrow \infty}\left(\left(x_{-}^{\lambda} \tau_{n}(x)\right) * x_{+}^{\mu}, \phi\right)=N-\lim _{n \rightarrow \infty} I_{2}= & \left(B(-\lambda-\mu-1, \mu+1) x_{-}^{\lambda+\mu+1}\right. \\
& \left.+B(-\lambda-\mu-1, \lambda+1) x_{+}^{\lambda+\mu+1}, \phi\right) .
\end{aligned}
$$

On the other hand, we can follow a similar step to evaluate

$$
\begin{aligned}
\left(x_{-}^{\lambda} *\left(x_{+}^{\mu} \tau_{n}(x)\right), \phi\right)= & \left(y_{-}^{\lambda},\left(x_{+}^{\mu} \tau_{n}(x), \phi(x+y)\right)\right) \\
= & \int_{-n-n^{-n}}^{0}(-y)^{\lambda} \int_{a}^{b}(x-y)_{+}^{\mu} \tau_{n}(x-y) \phi(x) d x d y \\
= & \int_{a}^{b} \phi(x) \int_{-n-n^{-n}}^{-n}(-y)^{\lambda}(x-y)_{+}^{\mu} \tau_{n}(x-y) d y d x \\
& +\int_{a}^{b} \phi(x) \int_{-n}^{0}(-y)^{\lambda}(x-y)_{+}^{\mu} \tau_{n}(x-y) d y d x
\end{aligned}
$$

When $x<0$ and $-n \leq y \leq 0, \tau_{n}(x-y)=1$ by assuming $n>-a$. This implies that

$$
\begin{aligned}
\int_{-n}^{0}(-y)^{\lambda}(x-y)_{+}^{\mu} \tau_{n}(x-y) d y & =\int_{-n}^{x}(-y)^{\lambda}(x-y)^{\mu} d y \\
& =(-x)^{\lambda+\mu+1} \int_{-x / n}^{1} u^{-\lambda-\mu-2}(1-u)^{\mu} d u
\end{aligned}
$$

using the substitution $y=x u^{-1}$. This infers that

$$
N-\lim _{n \rightarrow \infty} \int_{-n}^{0}(-y)^{\lambda}(x-y)_{+}^{\mu} \tau_{n}(x-y) d y=B(-\lambda-\mu-1, \mu+1)(-x)^{\lambda+\mu+1} .
$$

When $x>0$ and $-n \leq y \leq 0$, we have

$$
\begin{aligned}
\int_{-n}^{0}(-y)^{\lambda}(x-y)_{+}^{\mu} \tau_{n}(x-y) d y & =\int_{x}^{x+n}(t-x)^{\lambda} t^{\mu} \tau_{n}(t) d t \\
& =\int_{x}^{n}(t-x)^{\lambda} t^{\mu} d t+\int_{n}^{n+n^{-n}}(t-x)^{\lambda} t^{\mu} \tau_{n}(t) d t
\end{aligned}
$$

On making the substitution $t=x u^{-1}$, we get

$$
\int_{x}^{n}(t-x)^{\lambda} t^{\mu} d t=x^{\lambda+\mu+1} \int_{x / n}^{1} u^{-\mu-\lambda-2}(1-u)^{\lambda} d u
$$


and

$$
N-\lim _{n \rightarrow \infty} x^{\lambda+\mu+1} \int_{x / n}^{1} u^{-\mu-\lambda-2}(1-u)^{\lambda} d u=B(-\lambda-\mu-1, \lambda+1) x^{\lambda+\mu+1}
$$

by the normalization in [9]. It follows similarly that

$$
\begin{aligned}
& \lim _{n \rightarrow \infty} \int_{a}^{b} \phi(x) \int_{-n-n^{-n}}^{-n}(-y)^{\lambda}(x-y)_{+}^{\mu} \tau_{n}(x-y) d y d x=0, \quad \text { and } \\
& \lim _{n \rightarrow \infty} \int_{n}^{n+n^{-n}}(t-x)^{\lambda} t^{\mu} \tau_{n}(t) d t=0 .
\end{aligned}
$$

Therefore,

$$
\begin{aligned}
N-\lim _{n \rightarrow \infty}\left(x_{-}^{\lambda} *\left(x_{+}^{\mu} \tau_{n}(x)\right), \phi\right)= & \left(B(-\lambda-\mu-1, \mu+1) x_{-}^{\lambda+\mu+1}\right. \\
& \left.+B(-\lambda-\mu-1, \lambda+1) x_{+}^{\lambda+\mu+1}, \phi\right) .
\end{aligned}
$$

In other words,

$$
x_{-}^{\lambda} x_{+}^{\mu}=B(-\lambda-\mu-1, \mu+1) x_{-}^{\lambda+\mu+1}+B(-\lambda-\mu-1, \lambda+1) x_{+}^{\lambda+\mu+1}
$$

for $\lambda, \mu>-1$ and $\lambda, \mu, \lambda+\mu+1 \neq 0,1,2, \cdots$.

By mathematical induction, we assume that equation (5) holds for $\mu>-1,-k<\lambda<-k+1$ and $\mu, \lambda+\mu+k \neq 0,1,2, \cdots$, where $k$ is some positive integer. This is certainly true when $k=1$. We are going to show that

$$
\begin{aligned}
x_{-}^{\lambda-1} \otimes x_{+}^{\mu} & =-\frac{\left(x_{-}^{\lambda} \otimes x_{+}^{\mu}\right)^{\prime}}{\lambda} \\
& =B(-\lambda-\mu, \mu+1) x_{-}^{\lambda+\mu}+B(-\lambda-\mu, \lambda) x_{+}^{\lambda+\mu} .
\end{aligned}
$$

Indeed, we have by Definition 2.2

$$
\begin{aligned}
& \left(\left(x_{-}^{\lambda} \otimes x_{+}^{\mu}\right)^{\prime}, \phi(x)\right)=-\left(x_{-}^{\lambda} \otimes x_{+}^{\mu}, \phi^{\prime}(x)\right) \\
& =N-\lim _{n \rightarrow \infty} \frac{1}{2}\left\{\left(\left(\left(x_{-}^{\lambda} \tau_{n}(x)\right) * x_{+}^{\mu}\right)^{\prime}, \phi(x)\right)+\left(\left(x_{-}^{\lambda} *\left(x_{+}^{\mu} \tau_{n}(x)\right)\right)^{\prime}, \phi(x)\right)\right\} .
\end{aligned}
$$

Clearly,

$$
\begin{aligned}
& \left(\left(x_{-}^{\lambda} \tau_{n}(x)\right) * x_{+}^{\mu}\right)^{\prime}=-\lambda\left(x_{-}^{\lambda-1} \tau_{n}(x)\right) * x_{+}^{\mu}+\left(x_{-}^{\lambda} \tau_{n}^{\prime}(x)\right) * x_{+}^{\mu} \\
& \left(x_{-}^{\lambda} *\left(x_{+}^{\mu} \tau_{n}(x)\right)\right)^{\prime}=-\lambda x_{-}^{\lambda-1} *\left(x_{+}^{\mu} \tau_{n}(x)\right) .
\end{aligned}
$$

It remains to prove that

$$
N-\lim _{n \rightarrow \infty}\left(\left(x_{-}^{\lambda} \tau_{n}^{\prime}(x)\right) * x_{+}^{\mu}, \phi(x)\right)=0
$$

since

$$
\begin{aligned}
& N-\lim _{n \rightarrow \infty} \frac{-\lambda}{2}\left\{\left(\left(x_{-}^{\lambda-1} \tau_{n}(x)\right) * x_{+}^{\mu}, \phi\right)+\left(x_{-}^{\lambda-1} *\left(x_{+}^{\mu} \tau_{n}(x)\right), \phi\right)\right\} \\
& =\left(-\lambda\left(x_{-}^{\lambda-1} x_{+}^{\mu}\right), \phi\right)
\end{aligned}
$$

using Definition 2.2.

Evidently,

$$
\left(\left(x_{-}^{\lambda} \tau_{n}^{\prime}(x)\right) * x_{+}^{\mu}, \phi(x)\right)=\int_{a}^{b} \phi(x) \int_{-n-n^{-n}}^{-n}(-y)^{\lambda} \tau_{n}^{\prime}(y)(x-y)^{\mu} d y d x
$$

since the support of $x_{-}^{\lambda} \tau_{n}^{\prime}(x)$ is contained in the interval $\left[-n-n^{-n},-n\right]$ and $n>-a>n^{-n}$ without loosing generality. 
Integrating by parts, we come to

$$
\begin{aligned}
& \int_{-n-n^{-n}}^{-n}(-y)^{\lambda} \tau_{n}^{\prime}(y)(x-y)^{\mu} d y \\
& =n^{\lambda}(x+n)^{\mu}+\int_{-n-n^{-n}}^{-n}\left[\lambda(-y)^{\lambda-1}(x-y)^{\mu}+\mu(x-y)^{\mu-1}(-y)^{\lambda}\right] \tau_{n}(y) d y=I_{1}+I_{2} .
\end{aligned}
$$

Choosing a positive integer $r$ greater than $\lambda+\mu$, we get by Taylor's formula

$$
I_{1}=n^{\lambda}(x+n)^{\mu}=n^{\lambda+\mu}\left(1+\frac{x}{n}\right)^{\mu}=n^{\lambda+\mu} \sum_{i=0}^{r} \frac{(\mu)_{i}}{i ! n^{i}} x^{i}+n^{\lambda+\mu} \frac{(\mu)_{r+1}\left(1+\frac{\zeta}{n}\right)^{\mu-r-1}}{(r+1) ! n^{r+1}} x^{r+1}
$$

where $\zeta$ is between 0 and $x$ and

$$
(\mu)_{i}= \begin{cases}1 & \text { if } i=0 \\ \mu(\mu-1) \cdots(\mu-i+1) & \text { if } i \geq 1\end{cases}
$$

Thus,

$$
\begin{aligned}
N-\lim _{n \rightarrow \infty}\left(I_{1}, \phi\right)= & N-\lim _{n \rightarrow \infty} n^{\lambda+\mu} \sum_{i=0}^{r} \frac{(\mu)_{i}}{i ! n^{i}} \int_{a}^{b} x^{i} \phi(x) d x \\
& +\lim _{n \rightarrow \infty} n^{\lambda+\mu} \frac{(\mu)_{r+1}}{(r+1) ! n^{r+1}} \int_{a}^{b}\left(1+\frac{\zeta}{n}\right)^{\mu-r-1} x^{r+1} \phi(x) d x=0
\end{aligned}
$$

by noting that $\lambda+\mu \neq 0,1,2, \cdots, r>\lambda+\mu$ and $\left(1+\frac{\zeta}{n}\right)^{\mu-r-1} x^{r+1} \phi(x)$ is bounded.

As for $I_{2}$, we can see that

$$
\lim _{n \rightarrow \infty} I_{2}=0
$$

by a similar argument mentioned above.

In summary, we derive that

$$
\begin{aligned}
x_{-}^{\lambda-1} \otimes x_{+}^{\mu} & =-\frac{\left(x_{-}^{\lambda} \otimes x_{+}^{\mu}\right)^{\prime}}{\lambda} \\
& =-\frac{1}{\lambda}\left(B(-\lambda-\mu-1, \mu+1) x_{-}^{\lambda+\mu+1}+B(-\lambda-\mu-1, \lambda+1) x_{+}^{\lambda+\mu+1}\right)^{\prime} \\
& =B(-\lambda-\mu, \mu+1) x_{-}^{\lambda+\mu}+B(-\lambda-\mu, \lambda) x_{+}^{\lambda+\mu}
\end{aligned}
$$

using the following identities

$$
\begin{aligned}
& \frac{d}{d x} x_{-}^{\lambda+\mu+1}=-(\lambda+\mu+1) x_{-}^{\lambda+\mu}, \\
& \frac{\lambda+\mu+1}{\lambda} B(-\lambda-\mu-1, \mu+1)=B(-\lambda-\mu, \mu+1), \\
& \frac{d}{d x} x_{+}^{\lambda+\mu+1}=(\lambda+\mu+1) x_{+}^{\lambda+\mu}, \\
& -\frac{\lambda+\mu+1}{\lambda} B(-\lambda-\mu-1, \lambda+1)=B(-\lambda-\mu, \lambda) .
\end{aligned}
$$

It follows from the above that equation (5) holds for $\mu>-1, \mu \neq 0,1,2, \cdots$ and $\lambda, \lambda+\mu \neq 0, \pm 1, \pm 2, \cdots$.

A parallel argument shows that equation (5) still holds for $-k<\mu<-k+1$ and $\lambda, \lambda+\mu \neq$ $0, \pm 1, \pm 2, \cdots$, where $k$ is some positive integer. This completes the proof of Theorem 2.3.

Remark 4 Theorem 2.3 was first proved by Fisher and Li in [13] using Definition 1.5 with complicated computations as many terms involve the product of $\tau_{n}(t)$ and $\tau_{n}(x-t)$ in general. 
Theorem 2.4 The commutative neutrix convolution $x_{-}^{\lambda} x_{+}^{s}$ exists and

$$
x_{-}^{\lambda} x_{+}^{s}=(-1)^{s+1} B(\lambda+1, s+1) x_{-}^{\lambda+s+1}
$$

for $\lambda \neq 0, \pm 1, \pm 2, \cdots$ and $s=0,1,2, \cdots$.

Proof. It follows from $\Gamma(-s)=\infty$ that

$$
B(-\lambda-s-1, \lambda+1)=0 .
$$

Applying the identity

$$
\Gamma(z) \Gamma(1-z)=\frac{\pi}{\sin z \pi}
$$

we have

$$
\begin{aligned}
B(-\lambda-s-1, s+1) & =\frac{\Gamma(-\lambda-s-1) \Gamma(s+1)}{\Gamma(-\lambda)} \\
& =\frac{(-1)^{s+1} \pi}{\Gamma(\lambda+s+2) \sin (\lambda+1) \pi} \cdot \frac{\Gamma(s+1)}{\Gamma(-\lambda)} \\
& =(-1)^{s+1} \frac{\Gamma(\lambda+1) \Gamma(s+1)}{\Gamma(\lambda+s+2)}=(-1)^{s+1} B(\lambda+1, s+1) .
\end{aligned}
$$

The rest follows exactly from the proof of equation (5) by restricting $\mu=s=0,1,2, \cdots$. This completes the proof of Theorem 2.4 .

Corollary 2.1 The following commutative neutrix convolutions exist and

$$
\begin{aligned}
& x_{+}^{\lambda} x_{-}^{s}=(-1)^{s+1} B(\lambda+1, s+1) x_{+}^{\lambda+s+1}, \\
& x_{-}^{\lambda} \otimes x^{s}=0, \\
& x_{+}^{\lambda} \otimes x^{s}=0
\end{aligned}
$$

for $\lambda \neq 0, \pm 1, \pm 2, \cdots$ and $s=0,1,2, \cdots$.

Proof. Equation (8) follows immediately on replacing $x$ by $-x$ in equation (7). The following two identities imply equation (9)

$$
\begin{aligned}
& x_{-}^{\lambda} * x_{-}^{s}=B(\lambda+1, s+1) x_{-}^{\lambda+s+1}, \\
& x^{s}=x_{+}^{s}+(-1)^{s} x_{-}^{s} .
\end{aligned}
$$

Equation (10) clearly follows on replacing $x$ by $-x$ in equation (9).

Theorem 2.5 The commutative neutrix convolution $x_{-}^{r} x_{+}^{s}$ exists and

$$
x_{-}^{r} x_{+}^{s}=B(r+1, s+1)\left[(-1)^{r+1} x_{+}^{r+s+1}+(-1)^{s+1} x_{-}^{r+s+1}\right]
$$

for $r, s=0,1,2, \cdots$.

Proof. It follows from reference [18] that

$$
B(-n, m)=(-1)^{m} B(m, n-m+1)
$$

for $m=1,2, \cdots, n$ and $n=1,2, \cdots$. The proof of Theorem 2.3 is still valid with $\lambda=r$ and $\mu=s$. This completes the proof of Theorem 2.5.

Corollary 2.2 The commutative neutrix convolution $x_{-}^{r} x^{s}$ exists and

$$
x_{-}^{r} x^{s}=(-1)^{r+1} B(r+1, s+1) x_{+}^{r+s+1}
$$

for $r, s=0,1,2, \cdots$. 
Proof. Since

$$
x^{s}=x_{+}^{s}+(-1)^{s} x_{-}^{s}
$$

we have

$$
\begin{aligned}
x_{-}^{r} \otimes\left(x_{+}^{s}+(-1)^{s} x_{-}^{s}\right) & =x_{-}^{r} \otimes x_{+}^{s}+x_{-}^{r} \otimes(-1)^{s} x_{-}^{s} \\
& =(-1)^{r+1} B(r+1, s+1) x_{+}^{r+s+1}
\end{aligned}
$$

by Theorem 2.5 .

By Corollary 2.2, we imply that

$$
x_{+}^{r} x^{s}=(-1)^{r+s+1} B(r+1, s+1) x_{-}^{r+s+1}
$$

for $r, s=0,1,2, \cdots$.

Corollary 2.3 The commutative neutrix convolution $x^{r} \otimes x^{s}$ exists and

$$
x^{r} \otimes x^{s}=-B(r+1, s+1)\left[x_{+}^{r+s+1}+(-1)^{r+s} x_{-}^{r+s+1}\right]
$$

for $r, s=0,1,2, \cdots$.

Proof. It follows immediately from Corollary 2.2.

Theorem 2.6 The commutative neutrix convolution $\frac{1}{1+e^{-a(x-c)}} \otimes \operatorname{sgn} x$ exists and

$$
\frac{1}{1+e^{-a(x-c)}} \otimes \operatorname{sgn} x=\frac{3 x}{2}+\frac{2}{a} \ln \left(1+e^{-a(x-c)}\right)-c
$$

where $a, c \in R$ and $a \neq 0$.

Proof. Consider

$$
\begin{aligned}
& \left(\frac{\tau_{n}(x)}{1+e^{-a(x-c)}} * \operatorname{sgn} x, \phi\right)=\left(\int_{-\infty}^{\infty} \frac{\tau_{n}(t) \operatorname{sgn}(x-t)}{1+e^{-a(t-c)}}, \phi\right) \\
& =\left(\int_{-n-n^{-n}}^{-n} \frac{\tau_{n}(t) d t}{1+e^{-a(t-c)}}+\int_{-n}^{x} \frac{d t}{1+e^{-a(t-c)}}-\int_{x}^{n} \frac{d t}{1+e^{-a(t-c)}}-\int_{n}^{n+n^{-n}} \frac{\tau_{n}(t) d t}{1+e^{-a(t-c)}}, \phi\right) \\
& =\left(I_{1}+I_{2}-I_{3}-I_{4}, \phi\right)
\end{aligned}
$$

by assuming $|x|<n$.

As for $I_{1}$, we can see that

$$
\frac{\tau_{n}(t)}{1+e^{-a(t-c)}}=\frac{e^{a(t-c)} \tau_{n}(t)}{1+e^{a(t-c)}}
$$

is bounded on the interval $\left[-n-n^{-n},-n\right]$. This implies that

$$
\lim _{n \rightarrow \infty}\left(I_{1}, \phi\right)=0 .
$$

Similarly, we derive that

$$
\lim _{n \rightarrow \infty}\left(I_{4}, \phi\right)=0 .
$$

Making the substitution $u=1+e^{-a(t-c)}$, we come to

$$
I_{2}-I_{3}=2 x+\frac{2}{a} \ln \left(1+e^{-a(x-c)}\right)-\frac{1}{a}\left[\ln \left(1+e^{-a(-n-c)}\right)+\ln \left(1+e^{-a(n-c)}\right)\right]
$$

It follows that

$$
N-\lim _{n \rightarrow \infty}-\frac{1}{a}\left[\ln \left(1+e^{-a(-n-c)}\right)+\ln \left(1+e^{-a(n-c)}\right)\right]=-c,
$$


which infers that

$$
N-\lim _{n \rightarrow \infty}\left(\frac{\tau_{n}(x)}{1+e^{-a(x-c)}} * \operatorname{sgn} x, \phi\right)=\left(2 x+\frac{2}{a} \ln \left(1+e^{-a(x-c)}\right)-c, \phi\right) .
$$

Following a similar step, we can show that

$$
N-\lim _{n \rightarrow \infty}\left(\frac{1}{1+e^{-a(x-c)}} * \operatorname{sgn} x \tau_{n}(x), \phi\right)=\left(x+\frac{2}{a} \ln \left(1+e^{-a(x-c)}\right)-c, \phi\right) .
$$

This completes the proof of Theorem 2.6.

From the above computation, we also get for $a, c \in R$ and $a \neq 0$

$$
\begin{aligned}
& \frac{1}{1+e^{-a(x-c)} \circledast \operatorname{sgn} x}=2 x+\frac{2}{a} \ln \left(1+e^{-a(x-c)}\right)-c, \\
& \operatorname{sgn} x \circledast \frac{1}{1+e^{-a(x-c)}}=x+\frac{2}{a} \ln \left(1+e^{-a(x-c)}\right)-c .
\end{aligned}
$$

\section{Fractional Calculus of Distributions}

In this section, we will use the new convolutions of distributions presented in section 2 to define fractional integrals and derivatives of some distributions without bounded support or support contained in $R^{+}$. We obtain a number of interesting and new results which are not achievable in the classical sense.

Let $\mathcal{D}^{\prime}\left(R^{+}\right)$be the subspace of $\mathcal{D}^{\prime}$ with support contained in $R^{+}$. It follows from [8] and [9] that $\Phi_{\lambda}=\frac{x_{+}^{\lambda-1}}{\Gamma(\lambda)} \in \mathcal{D}^{\prime}\left(R^{+}\right)$is an entire function of $\lambda$ on the complex plane, and

$$
\left.\frac{x_{+}^{\lambda-1}}{\Gamma(\lambda)}\right|_{\lambda=-n}=\delta^{(n)}(x), \quad \text { for } \quad n=0,1,2, \cdots
$$

For the functional $\Phi_{\lambda}=\frac{x_{+}^{\lambda-1}}{\Gamma(\lambda)}$, the derivative formula is simpler than that for $x_{+}^{\lambda}$. In fact,

$$
\frac{d}{d x} \Phi_{\lambda}=\frac{d}{d x} \frac{x_{+}^{\lambda-1}}{\Gamma(\lambda)}=\frac{(\lambda-1) x_{+}^{\lambda-2}}{\Gamma(\lambda)}=\frac{x_{+}^{\lambda-2}}{\Gamma(\lambda-1)}=\Phi_{\lambda-1} .
$$

Let $\lambda$ and $\mu$ be arbitrary complex numbers. Then it is easy to show

$$
\Phi_{\lambda} * \Phi_{\mu}=\Phi_{\lambda+\mu}
$$

by equation (12), without any help of analytic continuation mentioned in all current books.

Let $\lambda$ be an arbitrary complex number and $g(x)$ be the distribution concentrated on $x \geq 0$. We define the primitive of order $\lambda$ of $g$ as the convolution in the distributional sense [8]

$$
g_{\lambda}(x)=g(x) * \frac{x_{+}^{\lambda-1}}{\Gamma(\lambda)}=g(x) * \Phi_{\lambda} .
$$

Note that the convolution on the right-hand side is well defined by Definition 1.2 since the supports of $g$ and $\Phi_{\lambda}$ are bounded on the same side.

For a given function, its classical Riemann-Liouville derivative or Caputo derivative [19], [20] and [21] may not exist in general [7], [22] and [23]. Even if they do, the Riemann-Liouville derivative and the Caputo derivative are not necessarily the same. However, if $g(x)$ is a distribution in $\mathcal{D}^{\prime}\left(R^{+}\right)$, then the case is different. Let $m-1<\operatorname{Re} \lambda<m \in Z^{+}$. From equation (2), we derive that

$$
\begin{aligned}
g_{-\lambda}(x) & =g(x) * \frac{x_{+}^{-\lambda-1}}{\Gamma(-\lambda)}=g(x) * \frac{d^{m}}{d x^{m}} \frac{x_{+}^{m-\lambda-1}}{\Gamma(m-\lambda)} \\
& =\frac{d^{m}}{d x^{m}}\left(g(x) * \frac{x_{+}^{m-\lambda-1}}{\Gamma(m-\lambda)}\right)=\frac{x_{+}^{m-\lambda-1}}{\Gamma(m-\lambda)} * g^{(m)}(x),
\end{aligned}
$$


which indicates there is no difference between the Riemann-Liouville derivative and the Caputo derivative of the distribution $g(x)$ (both exist clearly). Based on this fact, we only call the fractional derivative of distribution for brevity.

Equation (13) derives that if $g$ is a distribution concentrated on $R^{+}$, then

$$
\left(g * \Phi_{\lambda}\right) * \Phi_{\mu}=g *\left(\Phi_{\lambda} * \Phi_{\mu}\right)=g * \Phi_{\lambda+\mu} .
$$

Setting $\mu=-\lambda$ we see that differentiation and integration of the same order are mutually inverse processes. It follows from equation (15) that

$$
\frac{d^{\beta}}{d x^{\beta}}\left(\frac{d^{\gamma} g}{d x^{\gamma}}\right)=\frac{d^{\beta+\gamma} g}{d x^{\beta+\gamma}}
$$

for any $\beta$ and $\gamma$.

Replacing $\lambda$ by $-\lambda$ in equation (13), we get

$$
\frac{d^{\lambda}}{d x^{\lambda}}\left(\frac{x_{+}^{\mu-1}}{\Gamma(\mu)}\right)=\frac{x_{+}^{\mu-\lambda-1}}{\Gamma(\mu-\lambda)} .
$$

In particular, we have

$$
\begin{aligned}
& \frac{d^{\frac{1}{2}}}{d x^{\frac{1}{2}}} x_{+}=\frac{2 x_{+}^{\frac{1}{2}}}{\sqrt{\pi}}, \quad \frac{d^{-\frac{1}{2}}}{d x^{-\frac{1}{2}}} x_{+}=\frac{4 x_{+}^{1.5}}{3 \sqrt{\pi}}, \\
& \frac{d^{\frac{1}{2}}}{d x^{\frac{1}{2}}} \theta(x)=\frac{x_{+}^{-\frac{1}{2}}}{\sqrt{\pi}}, \quad \frac{d^{-\frac{1}{2}}}{d x^{-\frac{1}{2}}} \theta(x)=\frac{2}{\sqrt{\pi}} x_{+}^{0.5}, \\
& \frac{d^{0.25}}{d x^{0.25}} x_{+}^{-1.5}=\frac{\Gamma(-0.5)}{\Gamma(-0.75)} x_{+}^{-1.75}=\frac{-2 \sqrt{\pi}}{\Gamma(-0.75)} x_{+}^{-1.75} .
\end{aligned}
$$

Writing $\mu=-k$ in equation (16), where $k$ is a nonnegative integer, we come to

$$
\frac{d^{\lambda}}{d x^{\lambda}} \delta^{(k)}(x)=\frac{x_{+}^{-k-\lambda-1}}{\Gamma(-k-\lambda)} .
$$

In particular,

$$
\begin{gathered}
\frac{d^{\frac{1}{2}}}{d x^{\frac{1}{2}}} \delta(x)=\frac{x_{+}^{-1.5}}{\Gamma(-0.5)}=-\frac{x_{+}^{-1.5}}{2 \sqrt{\pi}}, \\
\frac{d^{\frac{1}{2}}}{d x^{\frac{1}{2}}} \delta^{\prime}(x)=\frac{x_{+}^{-2.5}}{\Gamma(-1.5)}=\frac{3 x_{+}^{-2.5}}{4 \sqrt{\pi}}
\end{gathered}
$$

using

$$
\Gamma(-1 / 2)=-2 \sqrt{\pi}, \quad \Gamma(-3 / 2)=4 \sqrt{\pi} / 3 .
$$

Setting $\mu-\lambda=-k$ in equation (16), where $k$ is a nonnegative integer, we have

$$
\frac{d^{\lambda}}{d x^{\lambda}}\left(\frac{x_{+}^{\lambda-k-1}}{\Gamma(\lambda-k)}\right)=\delta^{(k)}(x) .
$$

Let $\lambda$ be an arbitrary complex number and $H$ be a subspace of $\mathcal{D}^{\prime}$ given by

$$
H=\left\{g \in \mathcal{D}^{\prime} \mid g \propto \Phi_{\lambda} \text { exists }\right\} .
$$

Clearly, $H$ contains $\mathcal{D}^{\prime}\left(R^{+}\right)$as a proper subspace since Definition 2.2 generalizes Definition 1.2.

Let $g(x)$ be the distribution in $H$. We define the primitive of order $\lambda$ of $g$ as the convolution given by Definition 2.2 in the distributional sense

$$
g_{\lambda}(x)=g(x) \triangleq \frac{x_{+}^{\lambda-1}}{\Gamma(\lambda)}=g(x) \Leftarrow \Phi_{\lambda} .
$$


Note that the convolution on the right-hand side is well defined since $g$ is in $H$.

Thus equation (17) with various $\lambda$ will not only give the fractional derivatives, but also the fractional integrals of $g(x) \in H$ when $\lambda \notin Z$, and it reduces to integer-order derivatives or integrals when $\lambda \in Z$. We shall define the convolution

$$
g_{-\lambda}=g(x) \leftrightarrow \Phi_{-\lambda}
$$

as the fractional derivative of the distribution $g(x)$ with order $\lambda$, writing it as

$$
g_{-\lambda}=\frac{d^{\lambda}}{d x^{\lambda}} g
$$

for $\operatorname{Re} \lambda \geq 0$. Similarly, $\frac{d^{\lambda}}{d x^{\lambda}} g$ is interpreted as the fractional integral of order $\lambda$ if $\operatorname{Re} \lambda<0$.

Theorem 3.1

$$
\frac{d^{\lambda}}{d x^{\lambda}} x_{-}^{\mu}=\frac{1}{\Gamma(-\lambda)}\left[B(\lambda-\mu,-\lambda) x_{-}^{\mu-\lambda}+B(\lambda-\mu, \mu+1) x_{+}^{\mu-\lambda}\right]
$$

for $\mu-\lambda \neq 0,1,2, \cdots, \lambda, \mu, \lambda+\mu \neq 0, \pm 1, \pm 2, \cdots$.

Proof. Clearly, we have by Theorem 2.3

$$
\begin{aligned}
\frac{d^{\lambda}}{d x^{\lambda}} x_{-}^{\mu} & ==x_{-}^{\mu} \otimes \frac{x_{+}^{-\lambda-1}}{\Gamma(-\lambda)} \\
& =\frac{1}{\Gamma(-\lambda)}\left[B(\lambda-\mu,-\lambda) x_{-}^{\mu-\lambda}+B(\lambda-\mu, \mu+1) x_{+}^{\mu-\lambda}\right]
\end{aligned}
$$

for $\mu-\lambda \neq 0,1,2, \cdots, \lambda, \mu, \lambda+\mu \neq 0, \pm 1, \pm 2, \cdots$.

In particular,

$$
\frac{d^{0.5}}{d x^{0.5}} x_{-}^{0.75}=-\frac{1}{2 \sqrt{\pi}}\left[B(-0.25,-0.5) x_{-}^{0.25}+B(-0.25,1.75) x_{+}^{0.25}\right] .
$$

Remark 5 The previous studies on fractional derivatives of distributions in [5], [6], [7] and [8] are unable to give the above results since the support of $x_{-}^{\mu}$ is contained in $(-\infty, 0]$. By the way, there are Beta function calculators online from which we can find approximate distributions for fractional integrals and derivatives of generalized functions in $H$.

It follows that $\lambda$-order derivatives of distributions such as

$$
\begin{aligned}
& |x|^{\mu}=x_{+}^{\mu}+x_{-}^{\mu}, \\
& \operatorname{sgn} x \cdot|x|^{\mu}=x_{+}^{\mu}-x_{-}^{\mu}
\end{aligned}
$$

exist for some values of $\lambda$ and $\mu$ by Theorem 2.3. We leave them for interested readers.

Acknowledgments. This work is partially supported by the Natural Sciences and Engineering Research Council of Canada and Brandon University Research Grant. The authors are grateful to the reviewers for the careful reading of the paper along with several productive suggestions, which have improved its quality.

\section{References}

1. L. Cheng and C. Li, "A commutative product of distributions on $r^{m}$," Math. Nachr., vol. 151, pp. 345-355, 1991.

2. M. Aguirre and C. Li, "The distributional products of particular distributions," Appl. Math. Comput., vol. 187, pp. 20-26, 2007.

3. C. Li, "A review on the products of distributions," Mathematical Methods in Engineering (Springer), pp. 71-96, 2007.

4. - "The products on the unit sphere and even-dimension spaces," J. Math. Anal. Appl., vol. 305, pp. 97-106, 2005. 
5. C. Li, C. P. Li, B. Kacsmar, R. Lacroix, and K. Tilbury, "The abel integral equations in distribution," Advances in Analysis, vol. 2, pp. 88-104, 2017.

6. C. Li and C. P. Li, "Remarks on fractional derivatives of distributions," Tbilisi Mathematical Journal, vol. 10, pp. 1-18, 2017.

7. _ "On defining the distributions $(\delta)^{k}$ and $\left(\delta^{\prime}\right)^{k}$ by fractional derivatives," Appl. Math. Comput., vol. 246, pp. 502-513, 2014.

8. C. Li, "Several results of fractional derivatives in $D^{\prime}\left(R^{+}\right)$," Fractional Calculus and Applied Analysis, vol. 18, pp. 192-207, 2015.

9. I. M. Gel'Fand and G. E. Shilov, Generalized Functions. Academic Press, New York, 1964, vol. 1.

10. D. S. Jones, "The convolution of generalized functions," Quart. J. Math. Oxford Ser., vol. 24, pp. 145-163, 1973.

11. B. Fisher, "Neutrices and the convolution of distributions," Univ. u Novom Sadu Zb. Rad. Prirod. - Mat. Fak. Ser. Mat., vol. 17, pp. 119-135, 1987.

12. J. G. van der Corput, "Introdution to the neutrix calculus," J. Analyse Math., vol. 7, pp. 291-398, 1959-1960.

13. B. Fisher and C. Li, "A commutative neutrix convolution product of distributions," Univ. u Novom Sadu Zb. Rad. Prirod. - Mat. Fak. Ser. Mat., vol. 23, pp. 13-27, 1993.

14. A. Kilicman, "A comparison on the commutative neutrix convolution of distributions and the exchange formula," Czechoslovak Mathematical Journal, vol. 51, no. 3, pp. 463-471, 2001.

15. C. Li, "Several results on the commutative neutrix product of distributions," Integral transforms Spec. Funct., vol. 18 , pp. 559-568, 2007.

16. B. Fisher, "A result of the convolution of distributions," Proc. Edinburgh Math. Soc., vol. 19, pp. 393-395, 1975.

17. _ - "On defining the convolution of distributions," Math. Nachr., vol. 106, pp. 261-268, 1982.

18. B. Fisher and Y. Kuribayashi, "Neutrices and the beta function," Rostock. Math. Kolloq., vol. 32, pp. 56-66, 1987.

19. C. P. Li, D. Qian, and Y. Chen, "On riemann-liouville and caputo derivatives," Discrete Dynamics in Nature and Society, vol. 2011, article ID 562494, 15 pages.

20. C. P. Li and Z. Zhao, "Introduction to fractional integrability and differentiability," The European Physical Journal-Special Topics, vol. 193, pp. 5-26, 2011.

21. C. P. Li, F. Zhang, J. Kurths, and F. Zeng, "Equivalent system for a multiple-rational-order fractional differential system," Phil. Trans. R. Soc. A., vol. 371, 2013, article ID 20120156, 30 pages.

22. C. P. Li and W. Deng, "Remarks on fractional derivatives," Appl. Math. Comput., vol. 187, pp. 777-784, 2007.

23. A. A. Kilbas, H. M. Srivastava, and J. J. Trujillo, Theory and Applications of Fractional Differential Equations. Elsevier, New York, 2006. 